\title{
Diseño y validación del cuestionario «\#EvalEF» para conocer el proceso de evaluación desarrollado por los docentes de educación física Design and validation of «\#EvalEF» questionnaire to value assessment processes developed by physical education teachers
}

\author{
Maite Zubillaga-O lague, Laura Cañadas \\ Universidad Autónoma de Madrid (España)
}

\begin{abstract}
Resumen. Esta investigación tiene como finalidad diseñar y validar un cuestionario para conocer la autopercepción de los docentes de educación física sobre los procesos de eval uación que llevan a cabo en su aula. Parael diseño se real izó unarevisión bibliográfica en torno al objeto de estudio. Posteriormente se real izó la val idación de contenido contando con la participación de 5 jueces expertos. Para valorar la fiabilidad del cuestionario se contó con 469 docentes de educación física en activo. Se comprobó la consistencia interna de la escala global y de cada una de sus dimensiones. Se realizó un análisis exploratorio para conocer la estructura latente de la escala y este se ratificó a través de un análisis factorial confirmatorio. La información se recogió duranteel curso 2019/2020. Seempleó el programaSPSS v. 22 y SPSSAmosv.26. El cuestionario final estácompuesto por 81 ítems de respuesta cerrada (Likert 1-6). Los resultados muestran la fiabilidad de la escala $(\alpha=.94)$. El análisisfactorial exploratorio muestralaexistencia de 6 factores, estructura que quedó posteriormente confirmada(RMSEA $=.08$ ). Por tanto, el cuestionario desarrollado es un instrumento válido y fiable para conocer la percepción de los docentes de educación física sobre los procesos de evaluación que llevan a cabo en su aula.
\end{abstract}

Palabras clave: Cuestionario, Diseño, Validación, Evaluación, Educación Física.

\begin{abstract}
The aim of this research is to design and validate a questionnaire to know the self-perception of physical education teachers about the assessment processes they carry out in their classroom. For the design, a bibliographic review was conducted about the object of study. Afterwards, the content validation was carried out with the participation of 5 expert judges. In order to assess the reliability of the questionnaire, 469 active physical education teachers participated. The internal consistency of the global scale was assessed. Then, an exploratory analysis was conducted to know the latent structure of the scale which was ratified through a confirmatory factor analysis. Information was gathered during 2019/ 2020 academic year. SPSS v. 22 and SPSSA mos v. 26 programs were used. The final questionnaire is composed of 81 closed-response items (Likert 1-6). The results show the reliability of the scale $(\alpha=.94)$. The exploratory factor analysis shows the existence of 6 factors, a structure that was later confirmed (RMSEA $=.08$ ). Therefore, the developed questionnaire is a valid and reliable instrument to know the perception of physical education teachers about the assessment processes they carry out in their classroom.
\end{abstract}

Key words: Q uestionnaire, Design, Validation, Assessment, Physical Education.

\section{Introducción}

La evaluación es un elemento determinante en el proceso de enseñanza y aprendizaje que ha sufrido una importante evolución en lo referente a su concepción y finalidades. Tradicionalmente este proceso ha sido entendido como la certificación del aprendizaje del alumnado al finalizar un periodo formativo a través de una calificación (Blázquez-Sánchez, 2017; López-Pastor, 2017). Ú nicamente importaba la nota obtenida por el alumnado con objeto de establecer un ranking en función de estas (Abdullah-Alotaibi, 2019; Canales, 2007). Sin embargo, las diferentes corrientes educativas que

Fecha recepción: 23-12-20. Fecha de aceptación: 06-03-21 Maite Zubillaga O lague

maite.zubillaga@ estudiante.uam.es surgieron a finales del s. XIX y principios del s. XX, trajeron un cambio en la concepción sobre ese proceso de evaluación. Entre las diferentes corrientes que emergen destaca la denominada evaluación formativa (Herrero-González, Manrique-Arribas y López-Pastor, 2021). Esta forma de concebir la evaluación la entiende como un elemento integrado e intrínseco al proceso de enseñanza y aprendizaje que busca la mejora de este (Cañadas, 2020; López-Pastor, 2013). Así, a la evaluación se le atribuye una triple finalidad: (i) la búsqueda de un mayor y mejor aprendizaje del alumnado; (ii) que el profesorado pueda recoger información que le permita la mejora de su enseñanza y; (iii) la mejora del propio proceso (Chngy Lund, 2018; Chrónín y Cosgrave, 2013; López-Pastor et al., 2007; Rodríguez-García, 2006).

Esta evolución en la concepción de la evaluación se ha dado de forma generalizada en todo el ámbito educa- 
tivo y en los diferentes niveles formativos. Concreta mente, en la asignatura de educación física (EF), desde esa visión más tradicional, la evaluación estaba ligada al rendimiento físico del alumnado y a la medición del cuerpo, centrándose en medir aspectos vinculados a la condición física y ejecución técnica de habilidades deportivas (López-Pastor, 2006a). Principalmente se empleaban los test de condición física, relegando a un segundo plano el valor educativo de la asignatura (Barrientos-Hernán, López-Pastor y Pérez-Brunicardi, 2019). La nueva perspectiva que emerge busca cambiar esta visión y finalidad de la EF otorgándole un valor a la asignatura que va más allá del entrenamiento del cuerpo. Se plantea un modelo de evaluación centrado en el proceso de enseñanza y aprendizaje, integrado en la práctica docente y en las tareas desarrolladas en el aula, que produzca conexiones entre lo aprendido y lo que hay que aprender, que implique diversidad de instrumentos de evaluación y, que fomente la participación del alumnado (Díaz-Lucea, 2012; López-Pastor, 2005; 2006b).

Este continuo entre ambos extremos: evaluación como certificación de un aprendizaje y evaluación como elemento para la mejora constante del proceso de enseñanza y aprendizaje aún se mantiene vigente, no habiendo ocurrido un cambio total en la forma de desa rrollar la evaluación en el contexto educativo, y específicamente, en la EF (Calatayud, 2021). Los estudios realizados en la asignatura de EF muestran que la tradición sumativa de la evaluación influye fuertemente en la acción evaluativa del profesorado (Chng y Lund, 2018; Gil-Flores y García] iménez, 2014; RodríguezNegro y Zulaika, 2016). Entre los estudios que analizan los procesos evaluativos que se desarrollan en el ámbito escolar, prevalecen los estudios de carácter cualitativo y experimental en los que se estudian la influencia de la evaluación tradicional y el impacto de integración de la evaluación formativa en la enseñanza (ChaverraFernández y Hernández Álvarez, 2019; JiménezJiménez y Navarro-Adelantado, 2008; Ní-Chróinín y Cosgrave, 2013). Estos estudios se centran en mostrar los beneficios y limitaciones de integrar prácticas de evaluación formativa en el proceso deenseñanzay aprendizaje sin analizar los procesos de evaluación llevados a cabo por los docentes de EF en su totalidad.

En el contexto de la formación inicial del profesora do de EF existen cuestionarios válidos y fiables para va lorar los procesos de evaluación llevados a cabo por el profesorado universitario (Castejón-O liva, Santos-Pastor y Palacios-Picos, 2015; De la O rden Hoz y Pimien-
taPrieto, 2016; López-Pastor y Palacios-Picos, 2015), sin embargo, esta situación es muy diferente en el contexto de la EF. Son muy pocas las investigaciones que recogen información en torno a la eval uación en la asignatura de EF que lo hagan a través de un cuestionario (Gil-Flores y García-Jiménez, 2014; González-Palacio, ChaverraFernández, Bustamante-Castillo yToro-Suaza, 2021; Rodríguez-N egro y Zulaika 2016), sin ser, además, estos datos homogéneosy contrastables entreellos. Por tanto, parece existir una debilidad metodológica en lo referente a la constatación de los procesos de evalua ción en EF al no encontrar un instrumento válido y fia ble en español que permita recoger información sobre la percepción de un número elevado de docentes espa ñoles. Por ello, el objetivo del presente estudio es diseñar y validar un cuestionario para conocer la autopercepción de los docentes de EF sobre los procesos de evaluación que llevan a cabo en su aula.

\section{Método}

\section{Población y Muestra}

La muestra estuvo compuesta por 469 docentes de EF del territorio nacional español. El muestreo fue al ea torio, incidental, no probabilístico y se ajusta a los parámetros establecidos para el estudio de un instrumento donde se requiere que al menos haya cinco sujetos por ítem y un número máximo de diez (Cervantes, 2005). LaTabla 1 y laTabla 2 recogen las características de los participantes.

\begin{tabular}{|c|c|c|c|c|}
\hline \multicolumn{2}{|c|}{ Variable } & Media(DT) & Max & Min \\
\hline \multicolumn{2}{|l|}{ Edad } & $41.7(9.5)$ & 69 & 22 \\
\hline \multicolumn{2}{|c|}{ Experiencia docente } & $15.38(9.93)$ & 40 & 0 \\
\hline \multicolumn{2}{|c|}{ Horas de For mación en Evaluación } & $1426(246.7)$ & 3000 & 0 \\
\hline \multicolumn{5}{|c|}{ Tabla 2.} \\
\hline \multicolumn{5}{|c|}{ Características de la muestra de la investigación } \\
\hline \multirow{2}{*}{\multicolumn{3}{|c|}{ Variable }} & $\mathrm{n}$ & $\%$ \\
\hline & & & 298 & 63.5 \\
\hline \multirow[t]{2}{*}{ Sexo } & Mujer & & 171 & 36.5 \\
\hline & Público & & 357 & 76.1 \\
\hline \multirow[t]{2}{*}{ Centro escolar } & Concertado & & 100 & 21.3 \\
\hline & Privado & & 12 & 2.6 \\
\hline \multirow{3}{*}{ Nivel Educativo } & Educación & & 236 & 50.3 \\
\hline & Educación & aria 0 bligatoria & 219 & 45.7 \\
\hline & Formación & onal & 14 & 3.0 \\
\hline \multirow{4}{*}{ Titulación Académica } & Grado en M & en Educación Física & 220 & 46.9 \\
\hline & Grado en C & & 122 & 26 \\
\hline & Doble Grac & agisterio en EP y CAFyD & 115 & 24.5 \\
\hline & 0 tros & & 12 & 2.4 \\
\hline \multirow{3}{*}{ Mayor Grado Académico } & Grado o eq & & 226 & 48.2 \\
\hline & Postgrado & & 227 & 48.4 \\
\hline & Doctorado & & 16 & 3.4 \\
\hline
\end{tabular}

\section{Instrumento}

El instrumento empleado para esta investigación es un cuestionario diseñado ad hoc compuesto por 81 ítems divididos en 13 dimensiones de respuesta cerrada esca la tipo Likert con seis niveles de respuesta, un primer 
apartado donde se recoge la información identificativa de los participantes y un segundo apartado donde se les pregunta por la formación recibida en evaluación.

\section{Diseño}

Se llevó a cabo una investigación cuantitativa con tres fases: (i) diseño del cuestionario; (ii) validación del contenido del cuestionario diseñado ad hoc; y (iii) análisis de la fiabilidad del cuestionario tras aplicarlo a una muestra de docentes de EF del territorio nacional.

\section{Diseño del cuestionario}

El proceso de creación del cuestionario sigue los procedimientos propuestos por Carretero-Dios y Pérez (2007). El diseño del cuestionario se realizó tras una revisión bibliográfica sobre aspectos relacionados con la temática objeto de estudio (Castejón-O liva, Santos-Pastor y Palacios-Picos, 2015; De la O rden Hoz y Pimienta-Prieto, 2016; Garrido-Guzmán, Zagalaz, Torres y Romero, 2010; Gil-Flores y García-Jiménez, 2014; González-Palacio, Chaverra-Fernández, BustamanteCastillo y Toro-Suaza, 2021; López-Pastor y Palacios-
Picos, 2015; Rodríguez-N egro y Zulaika, 2016). Parala construcción setuvieron en cuentalos componentesque forman parte del proceso de evaluación: (i) finalidad (para qué evaluar); (ii) qué evaluar; (iii) por qué evaluar; (iv) cómo evaluar (instrumentos, técnicas y procedimientos); (v) momentos (cuándo evaluar); (vi) participantes (quien evalúa y a quien se evalúa); y (vii) calificación. Teniendo en cuenta estos apartados, se han establecido 13 dimensiones junto con un primer apartado donde se recoge la información identificativa de los participantes y un segundo apartado donde se les pregunta por la formación recibida en evaluación.

\section{Validez del contenido}

Posteriormente, se procedió a la validación de contenido mediante el juicio crítico de cinco expertos en el área de evaluación formativa y compartida en EF. Los expertos se seleccionaron de manera incidental. Tres de ellos son docentes titulares de Universidad y dos catedráticos de U niversidad, pertenecientes a cinco universidades del territorio nacional. Suman más de 75 años de experiencia docente en la universidad y cuen-

Tabla 3.

Cuestionario: Dimensiones, ítems y forma de respuesta

\begin{tabular}{|c|c|}
\hline Dimensión & Items \\
\hline Identificación & $\begin{array}{l}\text { I. Edad; II. Sexo; III. Años de experiencia como profesor/ a; IV. Ejerzo la docencia en un centro de carácter; V. Provincia donde ejerzo la docencia; VI. Localidad donde } \\
\text { ejerzo la docencia; VII. Situación laboral actual, VIII. Soy profesor de Educación Física en; IX. Titulación académica (grado o equival ente); X. M ayor grado académico } \\
\text { obtenido }\end{array}$ \\
\hline Formación & 1.1. Indique donde ha recibido formación; 1.2. Indique número de horas aproximadas de formación recibida \\
\hline $\begin{array}{l}\text { Finalidad. Likert 1-6 Grado } \\
\text { acuerdo. }\end{array}$ & $\begin{array}{l}2.1 \text { M ejorar el proceso de enseñanza y aprendizaje; } 2.2 \text {. Calificar al alumnado en función de los resultados obtenidos; } 2.3 \text {. Valorar el grado de consecución de los } \\
\text { objetivos propuestos por parte del alumnado; } 2.4 \text { M odificar mi enseñanza (relación con el alumnado, organización de las clases, explicaciones adecuadas, dominio } \\
\text { de la materia, etc.); } 2.5 \text {. Adaptar el proceso de enseñanza a las capacidades y ritmos del alumnado; } 2.6 \text {. Informar al alumnado de los aspectos a mejorar para que } \\
\text { alcancen los objetivos de aprendizaje propuestos; } 2.7 \text {. Medir el desempeño (condición física, bagaje motor) o rendimiento (velocidad de carrera, equilibrio, fuerza, } \\
\text { flexibilidad, etc.) del al umnado }\end{array}$ \\
\hline $\begin{array}{l}\text { Programación. Likert 1-6 Grado o } \\
\text { acuerdo. }\end{array}$ & $\begin{array}{l}\text { 3.1. Selecciono el instrumento/ s de evaluación que emplearé para registrar el desempeño del alumnado; 3.2. Establezco criterios de evaluación específicos para } \\
\text { cada U nidad Didáctica partiendo de los elementos curriculares establecidos para la etapa y curso; 3.3. Diseño y secuencio criterios de evaluación acordes a las } \\
\text { diferentes situaciones de enseñanza-aprendizaje que voy a plantear; 3.4. Construyo los criterios de evaluación de forma consensuada con mi alumnado; } 3.5 \text {. } \\
\text { Comparto los criterios de eval uación con mi alumnado al comienzo de la Unidad Didáctica }\end{array}$ \\
\hline $\begin{array}{l}\text { Evaluación Motricidad. Likert } 1-6 \\
\text { Grado de acuerdo. }\end{array}$ & $\begin{array}{l}\text { 4.1. Evalúo la correcta ejecución técnica (gestos/ formas propias de cada modalidad deportiva) de las habilidades deportivas; 4.2. Evalúo la utilización de } \\
\text { elementos tácticos (individuales o colectivos/ de cooperación y de oposición) en situaciones de juego; 4.3. Evalúo la aplicación del reglamento (conocimiento, } \\
\text { aplicación, utilización, etc.) en situaciones de juego; 4.4. Evalúo el estado de la condición física del alumnado; } 4.5 \text {. Evalúo la resolución de problemas motrices } \\
\text { (habilidades y destrezas motrices básicas, específicas, deportivas, etc.); 4.6. Evalúo la capacidad del alumnado para realizar creaciones artísticas y expresivas; } 4.7 \text {. } \\
\text { Evalúo la ejecución de técnicas de bailes y/ o danzas; 4.8. Evalúo la capacidad del alumnado para realizar actividades en el medio natural; } 4.9 \text {. Evalúo si el } \\
\text { alumno/ a conoce y respeta los hábitos higiénicos y de salud en la práctica de actividad física }\end{array}$ \\
\hline $\begin{array}{l}\text { Evaluación Conceptos. Likert 1-6 } \\
\text { Grado de acuerdo. }\end{array}$ & $\begin{array}{l}\text { 5.1. Evalúo la capacidad del alumnado para memorizar hechos o conceptos; } 5.2 \text {. Evalúo la capacidad del alumnado para utilizar los conocimientos teóricos en la } \\
\text { práctica; } 5.3 \text {. Evalúo la capacidad del alumnado para realizar juicios de valor sobre los contenidos trabajados; } 5.4 \text {. Evalúo la capacidad del alumnado para } \\
\text { comprender los contenidos trabajados }\end{array}$ \\
\hline $\begin{array}{l}\text { Evaluación Actitudes. Likert 1- } \\
\text { Grado de acuerdo. }\end{array}$ & $\begin{array}{l}\text { 6.1. Evalúo la disposición del alumnado para realizar las actividades propuestas; 6.2. Evalúo las actitudes (aceptación, respeto, interacción, etc.) hacia sus } \\
\text { compañeros/ as, hacia el profesorado y hacia el material; } 6.3 \text {. Evalúo el cumplimiento de las normas en clase; } 6.4 \text {. La cooperación con los compañeros; } 6.5 \text {. La } \\
\text { capacidad de liderazgo }\end{array}$ \\
\hline Momentos. Likert 1-6 Frecuencia. & 7.1. Al inicio del proceso de enseñanza-aprendizaje; 7.2. A lo largo del proceso de enseñanza-aprendizaje; 7.3. Al final del proceso de enseñanza-aprendizaje \\
\hline $\begin{array}{l}\text { Evaluación Inicial. Likert 1-6 Grado \& } \\
\text { de acuerdo. }\end{array}$ & $\begin{array}{l}\text { 8. 1. Conocer el nivel (condición y capacidad física) de partida del alumnado; 8.2. Efectuar un primer diagnóstico acerca de las necesidades, carencias y problemas; } \\
\text { 8.3. Adaptar lo programado al nivel inicial del alumnado; 8.4. Para categorizar y agrupar a los al umnos/ as según su nivel de partida }\end{array}$ \\
\hline $\begin{array}{l}\text { Evaluación Proceso. Likert 1-6 Grac } \\
\text { de acuerdo. }\end{array}$ & $\begin{array}{l}\text { 9.1. Regular la propia práctica docente a lo largo del proceso; } 9.2 \text {. Recoger información sobre el desempeño del alumnado; } 9.3 \text {. Dar información al alumnado } \\
\text { sobre cómo lo está haciendo en relación con lo esperado; } 9.4 \text {. Dar información al alumnado para mejorar; } 9.5 \text {. Comprobar si el ritmo de aprendizaje se ajusta a lo } \\
\text { previsto; } 9.6 \text {. Proponer nuevas actividades que den respuesta a las necesidades del alumnado; } 9.7 \text {. Establecer calificaciones parciales a lo largo del proceso }\end{array}$ \\
\hline $\begin{array}{l}\text { Evaluación final. Likert 1-6 Grado de } \\
\text { acuerdo }\end{array}$ & $\begin{array}{l}\text { 10.1. Realizar un bal ance global de los aprendizajes en función de los objetivos generales } \\
\text { 10.2. Establecer una calificación final; } 10.3 \text {. O bservar el grado de adqui sición de las competencias establecidas; } 10.4 \text {. Comprobar si ha habido una mejora r } \\
\text { en comparación con el nivel de partida del alumnado }\end{array}$ \\
\hline Instrumentos. Likert 1-6 Frecuencia & $\begin{array}{l}\text { 11.1. Hoja de observación; 11.2. Fichas de seguimiento; 11.3. Diario de clase; 11.4. Cuestionarios con escala de opinión; 11.5. Rúbricas de evaluación; } 11.6 . \\
\text { Lista de control; 11.7. Portafolios; 11.8. Asamblea; 11.9. Examen; 11.10. Aplicaciones móviles (kahoot, plickers, etc); 11.11. Test de Condición física para medir } \\
\text { la resistencia (ejem. "Test de cooper", "Course Navette"); 11.12. Test de Condición Física para medir la velocidad (ejem. Carrera de } 50 \text { m); } 11.13 \text {. Test de } \\
\text { Condición física para medir la fuerza (ejem. Lanzamiento de balón medicinal); 11.14. Test de Condición física para medir la flexibilidad (ejem. "sit and reach"); } \\
\text { 11.15. Test motores (ejem. Equilibrio "flamingo"); 11.16. Test de psicomotricidad (ejem. M SCA); 11.17. Situaciones de juego deportivo contextualizadas }\end{array}$ \\
\hline $\begin{array}{l}\text { Participantes. Likert } 1-6 \text { grado } \\
\text { acuerdo }\end{array}$ & $\begin{array}{l}\text { 12.1. El alumnado se evalúa a sí mismo (autoevaluación); 12.2. El alumnado evalúa a sus compañeros/ as (coevaluación); 12.3. El profesor/ a de Educación física } \\
\text { evalúa a su alumnado; } 12.4 \text {. El profesor/ a de Educación Física se evalúa a sí mismo; } 12.5 \text {. El profesor/ a de Educación Física es eval uado por su alumnado; } 12.6 \text {. El } \\
\text { profesor/ a de Educación Física es eval uado por sus compañeros/ as de departamento u otros agentes externos al proceso; } 12.7 \text {. Se evalúa el proceso de enseñanza- } \\
\text { aprendizaje (las actividades propuestas, organización, metodología, etc.) }\end{array}$ \\
\hline $\begin{array}{l}\text { Base de calificación. Li } \\
\text { de acuerdo }\end{array}$ & $\begin{array}{l}\text { 13.1. Es el rendimiento mostrado en pruebas (test de condición física, exhibiciones de baile, expresión corporal, partidos, etc.,) realizados; 13.2. Es el esfuerzo } \\
\text { realizado por el al umno/ a; 13.3. Es el progreso del alumno/ a desde el comienzo hasta el final de la unidad didáctica }\end{array}$ \\
\hline $\begin{array}{l}\text { Proceso Calificación. Likert 1-6 grado } \\
\text { de acuerdo }\end{array}$ & $\begin{array}{l}\text { 14.1. La calificación la decide el profesor/a; 14.2. El alumnado se autocalifica, establece su propia nota (parcial o totalmente); 14.3. Se califica de forma dialogada } \\
\text { y consensuada, entre profesorado y al umnado (parcial o totalmente); 14.4. Se califica a partir de la autoevaluación (parcial o totalmente); } 14.5 \text {. Se califica a partir } \\
\text { de la coevaluación, entre compañeros/ as (parcial o totalmente) }\end{array}$ \\
\hline
\end{tabular}


tan con más de 100 publicaciones científicas sobre evaluación y EF. A los expertos se les pidió que valoraran el grado de suficiencia, coherencia, relevancia y claridad de cada uno de los ítems propuestos para la escala, siguiendo los indicadores propuestos por Pérez y Cuervo (2008). Se estableció una escala de 1 a 4, donde 1 corresponde a «no cumple el criterio» y a 4 «lo cumple en su totalidad». Tras la valoración del juicio de expertos, se eliminó una pregunta entera y 5 ítems de diversos bloques de preguntas. Por último, se sumaron dos ítems a otra de las preguntas. Finalmente, el cuestionario quedó compuesto por 81 ítems agrupados en 13 dimensiones más un primer bloque de preguntas para conocer las características generales del profesorado participante junto a un segundo bloque para conocer la formación recibida en evaluación. Las dimensiones e ítems finales que componen el cuestionario se encuentran en laTabla 3. U na vez diseñada esta última versión del cuestionario se transcribió en la plataforma Google Forms. Laspreguntas se contestaban con una escala Likert con 6 niveles de respuesta siendo 1. Muy en desacuerdo/ nunca y 6. Muy de acuerdo/ Siempre (a excepción de la primer y segundo apartado, por tratarse de información relacionada con sus características y experiencias). En laTabla 3 se muestran los ítems que componen cada dimensión del cuestionario junto con la forma de respuesta por cada dimensión.

\section{Análisis de datos}

Se realizó un análisis factorial exploratorio (AFE) a través de un análisis de componentes principales (ACP) con rotación Varimax para identificar la estructura la tente de la escala. Para val orar la adecuación de los diferentes componentes estructurales de la escala se toma ron como referencia los val ores de Kaiser-M ayer-O Ikin (KMO) y la prueba de Esfericidad de Bartlett. Para ello se empleó el programa SPSS v.22. Posteriormente, se realizó un análisis factorial confirmatorio (AFC) para confirmar la adecuación de las dimensiones extraídas para la escala. Se excluyó del análisis las dimensiones relacionadas con los datos de identificación de los participantes y de la de formación en evaluación recibida. Para realizar el análisis se utilizó el programa SPSS AMOS. V26. Se empleó el método de estimación de máxima verosimilitud (ML), los índices absolutos de chi cuadrado $\left(x^{2}\right)$, el RM SEA (Root M ean Square, Residual o media de residuos estandarizados) y el CFI (Comparative fit o ajuste comparado).

Para analizar la consistencia interna de la escala se utilizó el Alpha de Cronbach, que también se calculó
Tabla 4.

A nálisis Factorial de la escala, matriz de componentes

\begin{tabular}{lccccccc}
\hline \multicolumn{1}{c}{ Ítems } & \multicolumn{3}{c}{ Componente } & Factor \\
\cline { 2 - 6 } & 1 & 2 & 3 & 4 & 5 & 6 & \\
Item.6.2. & .858 & & & & & \\
İtem.6.4. & .829 & & & & & \\
İtem.6.3 & .824 & & & & & \\
Ítem.6.1 & .796 & & & & & \\
Ítem.4.9 & .724 & & & & & \\
İtem.2.1 & .691 & & & & & \\
İtem.2.5. & .664 & & & &
\end{tabular}

İtem.2.6. $\quad .663$

Ítem.4.5. $\quad .658$

Ítem.3.2. $\quad .655$

$\begin{array}{lll}\text { Item.2.4. } & .649 & \text { (V1) Finalidades, programación y } \\ \text { Ittem.4.6. } & .647 & \text { contenidos con perspectiva formativa }\end{array}$

Ítem.2.3. $\quad .613$

$\begin{array}{ll}\text { Ítem.5.4. } & .594 \\ \text { Ítem.3.3. } & .593\end{array}$

Ítem.3.1. $\quad .535$

Ítem.4.3. $\quad .5318$

Ítem.5.3. $\quad .488$

Ítem.4.8. $\quad .452$

$\begin{array}{ll}\text { Item.3.5. } & .445 \\ \text { Ítem.6.5. } & .352\end{array}$

$\begin{array}{ll}\text { Item.9.5 } & .705 \\ \text { Item } 9.4 & .701\end{array}$

$\begin{array}{ll}\text { Ítem.9.4 } & .701 \\ \text { Ítem.9.6 } & .698\end{array}$

Ítem.9.1 $\quad .696$

Item.9.3 $\quad .678$

Ítem.9.2 .642

$\begin{array}{ll}\text { Ítem.10.1 } & .637 \\ \text { Ítem.8.2 } & .614\end{array}$

$\begin{array}{ll}\text { Ítem.8.2 } & .614 \\ \text { Ítem.8.3 } & .585\end{array}$

Ítem.10.3 $\quad .567$

Item.10.4 $\quad .550$

Ítem.7.1 .442

Ítem.7.2

$\begin{array}{ll}\text { Ítem.8.1 } & .413 \\ \text { Îtem.12.4 } & .336\end{array}$

\begin{tabular}{ll} 
İtem.12.7 & .336 \\
\hline Item.14.4 & .335
\end{tabular}

$\begin{array}{ll}\text { Item.14.4 } & .832 \\ \text { İtem.14.2 } & .784\end{array}$

$\begin{array}{ll}\text { Item.14.2 } & .784 \\ \text { Ittem.14.5 } & .782\end{array}$

İtem.14.3

$\begin{array}{ll}\text { Ítem.12.1 } & .768 \\ \text { Ítem.12.2 } & .728\end{array}$

$\begin{array}{ll}\text { Ítem.12.2 } & .728 \\ \text { Ítem.12.5 } & .565\end{array}$

İtem.12.6 $\quad .481$

$\begin{array}{ll}\text { Item.14.1 } & -.475 \\ \text { Ítem.3.4 } & .467\end{array}$

$\begin{array}{ll}\text { Ítem.11.4 } & .461 \\ \text { Ítem.11.8 } & .623\end{array}$

\begin{tabular}{lr} 
Item.11.8 & .623 \\
\hline Item.4.1 & .600
\end{tabular}

$\begin{array}{ll}\text { Ítem. } 2.2 & .500 \\ \text { Ítem.10.2 } & .587 \\ & .537\end{array}$

Ítem.4.4

Ítem.2.7 $\quad .531$

$\begin{array}{ll}\text { Item.4.7 } & .520 \\ \text { İtem.5.1 } & .514\end{array}$

$\begin{array}{ll}\text { Item.5.1 } & .514 \\ \text { İtem.13.1 } & .506\end{array}$

$\begin{array}{ll}\text { Ítem.11.9 } & .461 \\ \text { İtem.5.2 } & .416\end{array}$

$\begin{array}{ll}\text { Ítem.8.4 } & .411 \\ \text { Ítem.9.7 } & .623\end{array}$

Item.11.12

Ítem.11.14

Ítem.11.5

Ítem.11.13

Ítem.11.11

Ítem.11.16

Ítem.11.17

Item.13.2

Ítem.11.7

İtem.11.10

Ítem.11.3

İtem.11.6

Item.11.1

Ítem.11.2

por nivel educativo. Para valorar los resultados se ha tomado como referencia el valor propuesto por FríasN avarro (2019) y Nunnally (1978), por el que los val ores de $\alpha » .70$ se consideran como buenos. Se estimó el coeficiente de correlación entre el ítem, la escala (r) y el Alpha de Cronbach si se elimina el ítem de la escala 
Tabla 5.

Correlación elemento-escala y Alpha si se elimina el ítem

\begin{tabular}{|c|c|c|c|}
\hline Dimensión & Ítems & $\begin{array}{l}\text { r elemento } \\
\text { total }\end{array}$ & $\begin{array}{c}\alpha \text { si se elimina el } \\
\text { ítem }\end{array}$ \\
\hline \multirow{7}{*}{ Finalidad } & 2.1 Mejor ar el proceso de enseñanzay aprendizaje & .660 & .791 \\
\hline & 2.2. Calificar al alumnado en función de los resultados obtenidos & .428 & .831 \\
\hline & 2.3. Valorar el grado de consecución de los objetivos propuestos por parte del alumnado & .601 & .799 \\
\hline & 2.4. Modificar mi enseñanza (relación con el alumnado, organización de las clases, explicaciones adecuadas, dominio de la materia, etc.) & .642 & .793 \\
\hline & 2.5. Adaptar el proceso de enseñanza a las capacidades y ritmos del alumnado & .627 & .794 \\
\hline & 2.6. Informar al al umnado de los aspectos a mejorar para que alcancen los objetivos de aprendizaje propuestos & .722 & .782 \\
\hline & 2.7. Medir el desempeño (condición física, bagaje motor) o rendimiento (velocidad de carrera, equilibrio, fuerza, flexibilidad, etc.) del alumnado & .440 & .832 \\
\hline \multirow{5}{*}{ Programación } & 3.1. Selecciono el instrumento/ s de evaluación que emplearé para registrar el desempeño del alumnado & .521 & .717 \\
\hline & 3.2. Establezco criterios de evaluación específicos para cada Unidad Didáctica partiendo de los el ementos curriculares establecidos para la etapa y curso & 641 & 681 \\
\hline & 3.3. Diseño y secuencio criterios de evaluación acordes a las diferentes situaciones de enseñanza-aprendizaje que voy a plantear. & .683 & .661 \\
\hline & 3.4. Construyo los criterios de evaluación de forma consensuada con mi al umnado & .279 & .810 \\
\hline & 3.5. Compar to los criterios de eval uación con mi alumnado al comienzo de la Unidad Didáctica & .584 & .693 \\
\hline \multirow{9}{*}{ Motricidad } & 4.1. Evalúo la correcta ejecución técnica (gestos/ formas propias de cada modalidad deportiva) de las habilidades deportivas & .547 & .839 \\
\hline & 4.2. Evalúo la utilización de elementos tácticos (individuales o colectivos/ de cooperación y de oposición) en situaciones de juego. & 664 & .827 \\
\hline & 4.3. Evalúo la aplicación del reglamento (conocimiento, aplicación, utilización, etc.) en situaciones de juego & 618 & .831 \\
\hline & 4.4. Evalúo el estado de la condición física del alumnado & .447 & .852 \\
\hline & 4.5. Evalúo la resolución de problemas motrices (habilidades y destrezas motrices básicas, específicas, deportivas, etc.) & 654 & .830 \\
\hline & 4.6. Evalúo la capacidad del alumnado para real izar creaciones artísticas y expresivas & .620 & .832 \\
\hline & 4.7. Evalúo la ejecución de técnicas de bailes y/ 0 danzas & .648 & .828 \\
\hline & 4.8. Evalúo la capacidad del al umnado para real izar actividades en el medio natural & .472 & .847 \\
\hline & 4.9. Evalúo si el alumno/ a conoce y respeta los hábitos higiénicos y de salud en la práctica de actividad física & .541 & .839 \\
\hline \multirow{4}{*}{ Conceptos } & 5.1. Evalúo la capacidad del alumnado para memorizar hechos o conceptos & .313 & .826 \\
\hline & 5.2. Evalúo la capacidad del al umnado para utilizar los conocimientos teóricos en la práctica & .629 & .642 \\
\hline & 5.3. Evalúo la capacidad del al umnado para real izar juicios de valor sobre los contenidos trabajados & .642 & .638 \\
\hline & 5.4. Evalúo la capacidad del al umnado para comprender los contenidos trabajados & .656 & .641 \\
\hline \multirow{5}{*}{ Actitudes } & 6.1. Evalúo la disposición del alumnado para realizar las actividades propuestas & .823 & .844 \\
\hline & 6.2. Evalúo las actitudes (aceptación, respeto, interacción, etc.) hacia sus compañeros/ as, hacia el profesorado y hacia el material & .873 & .835 \\
\hline & 6.3. Evalúo el cumplimiento de las normas en clase & .859 & .836 \\
\hline & 6.4. La cooperación con los compañeros & .867 & .835 \\
\hline & 6.5. La capacidad de liderazgo & .404 & .962 \\
\hline \multirow{3}{*}{ Momentos } & 7.1. Al inicio del proceso de enseñanza-aprendizaje & .547 & .398 \\
\hline & 7.2. A lo largo del proceso de enseñanza-aprendizaje & .501 & .466 \\
\hline & 7.3. Al final del proceso de enseñanza aprendizaje & .344 & .656 \\
\hline & 8.1. Conocer el nivel (condición y capacidad física) de par tida del alumnado & .528 & .615 \\
\hline & 8.2. Efectuar un primer diagnóstico acerca de las necesidades, carencias y problemas & .607 & .586 \\
\hline InIClo & 8.3. Adaptar lo programado al nivel inicial del alumnado & .531 & .624 \\
\hline & 8.4. Para categorizar y agrupar a los alumnos/ as según su nivel de partida & .371 & .745 \\
\hline & 9.1. Regular la propia práctica docente a lo largo del proceso & .626 & .824 \\
\hline & 9.2. Recoger información sobre el desempeño del alumnado & .657 & .820 \\
\hline & 9.3. Dar infor mación al alumnado sobre cómo lo está haciendo en relación con lo esper ado & .709 & .813 \\
\hline Proceso & 9.4. Dar información al alumnado para mejorar & .735 & .812 \\
\hline & 9.5. Comprobar si el ritmo de aprendizaje se ajusta a lo previsto & .687 & .815 \\
\hline & 9.6. Proponer nuevas actividades que den respuesta a las necesidades del al umnado & .674 & .819 \\
\hline & 9.7. Establecer calificaciones parciales a lo lar go del proceso & .355 & .886 \\
\hline & 10.1. Realizar un bal ance global de los aprendizajes en función de los objetivos gener ales & .673 & .584 \\
\hline Final & 10.2. Establecer una cal ificación final & .365 & .772 \\
\hline rillal & 10.3. O bservar el grado de adquisición de las competencias establecidas & .610 & .605 \\
\hline & 10.4. Comprobar si ha habido una mejora notable en compar ación con el nivel de partida del alumnado & .472 & .683 \\
\hline & 11.1. Hoja de observación & .289 & .825 \\
\hline & 11.2. Fichas de seguimiento & .368 & .822 \\
\hline & 11.3. Diario de clase & .342 & .824 \\
\hline & 11.4. Cuestionarios con escala de opinión & .413 & .820 \\
\hline & 11.5. Rúbricas de eval uación & .261 & .827 \\
\hline & 11.6. Lista de control & .296 & .825 \\
\hline & 11.7. Portafolios & .375 & .821 \\
\hline & 11.8. Asamblea & .098 & .837 \\
\hline & 11.9. Examen & 311 & .825 \\
\hline Instrumentos & 11.10. Aplicaciones móviles (kahoot, plickers, etc) & .337 & .823 \\
\hline & 11.11. Test de Condición física para medir la resistencia (ejem. "Test de cooper”, "Course N avette”) & .632 & .806 \\
\hline & 11.12. Test de Condición Física para medir la velocidad (ejem. Carrera de $50 \mathrm{~m}$ ) & .683 & .803 \\
\hline & 11.13. Test de Condición física para medir la fuerza (ejem. Lanzamiento de balón medicinal) & .642 & .806 \\
\hline & 11.14. Test de Condición física para medir la flexibilidad (ejem. "sit and reach") & .675 & .804 \\
\hline & 11.15. Test motores (ejem. Equilibrio "flamingo") & 631 & .807 \\
\hline & 11.16. Test de psicomotricidad (ejem. MsCA) & .559 & .811 \\
\hline & 11.17. Situaciones de juego deportivo contextual izadas & .294 & .825 \\
\hline & 11.18. 0 tros & .259 & .828 \\
\hline & 12.1. El alumnado se evalúa a sí mismo (autoevaluación) & .629 & .721 \\
\hline & 12.2. El alumnado evalúa a sus compañeros/ as (coevaluación) & .574 & .737 \\
\hline & 12.3. El profesor/ a de Educación física evalúa a su alumnado & 198 & .791 \\
\hline Participantes & 12.4. El profesor/ a de Educación Física se evalúa a sí mismo & .539 & .744 \\
\hline & 12.5. El profesor/ a de Educación Física es eval uado por su alumnado & 656 & .713 \\
\hline & 12.6. El profesor/ a de Educación Física es eval uado por sus compañeros/ as de departamento u otros agentes externos al proceso & .418 & .777 \\
\hline & 12.7. Se evalúa el proceso de enseñanza-aprendizaje (las actividades propuestas, organización, metodología, etc.) & .508 & .747 \\
\hline & 13.1. Es el rendimiento mostrado en pruebas (test de condición física, exhibiciones de baile, expresión corporal, par tidos, etc., ) realizados & .146 & .686 \\
\hline Base calificación & 13.2. Es el esfuerzo realizado por el alumno/ a & .455 & .050 \\
\hline & 13.3. Es el progreso del alumno/ a desde el comienzo hasta el final de la unidad didáctica & .271 & .332 \\
\hline & 14.1. La calificación la decide el profesor/ a & -.355 & .874 \\
\hline & 14.2. El alumnado se autocalifica, establece su propia nota (parcial o totalmente) & .696 & .514 \\
\hline Procedimientos & 14.3. Se cal ifica de forma dial ogaday consensuada, entre profesorado y al umnado (parcial o total mente) & .590 & .557 \\
\hline Callitacion & 14.4. Se califica a partir de la autoevaluación (parcial o totalmente) & .748 & .481 \\
\hline & 14.5. Se califica a partir de la coevaluación, entre compañeros/ as (parcial o totalmente) & .684 & .513 \\
\hline
\end{tabular}

para valorar la adecuación de cada uno de los ítems. También se han valorado las correlaciones entre las diferentes dimensiones de la escala, a través de la correlación de Pearson. Se consideran valores significativos aquellos en los que $p<.05$.

\section{Resultados}

\section{Análisis Factorial Exploratorio}

La prueba de Kaiser-Mayer-O lkin (KMO) para la escala muestra un valor cercano al 1 (KMO $=.899)$, 10 
que determina que la realización de un análisis factorial esfactible. De manera adicional, la prueba deesfericidad de Bartlett muestraun nivel de significación de $p=.000$, con lo que también se confirma la viabilidad de realizar un análisis factorial. U na vez obtenidos los valores medios de cada constructo, se analiza la matriz de componentes para determinar la adecuación de los ítems pertenecientes a cada constructo o dimensión. En este caso se toma como criterio aceptar aquellos ítems cuyo valor sea superior a .30 (Gil-Flores y García-jiménez, 2014). Por tanto, la estructura latente de la escala propuesta está compuesta por 6 variables (Tabla 4).

\section{Análisis Factorial confirmatorio}

En la Figura 1 se presenta el modelo final de dimensiones e ítems para la escala a partir de las 6 dimensiones extraídas del AFE. Los resultados para el AFC mostraron un $x^{2}$ significativo $\left(x^{2}=14367 ; p<.000\right)$, por ello es necesario recurrir al resto de valores para conocer el ajuste de los datosal modelo teórico propuesto. El resto de los valores (RMSEA =.08; CFI=.50) presentan un
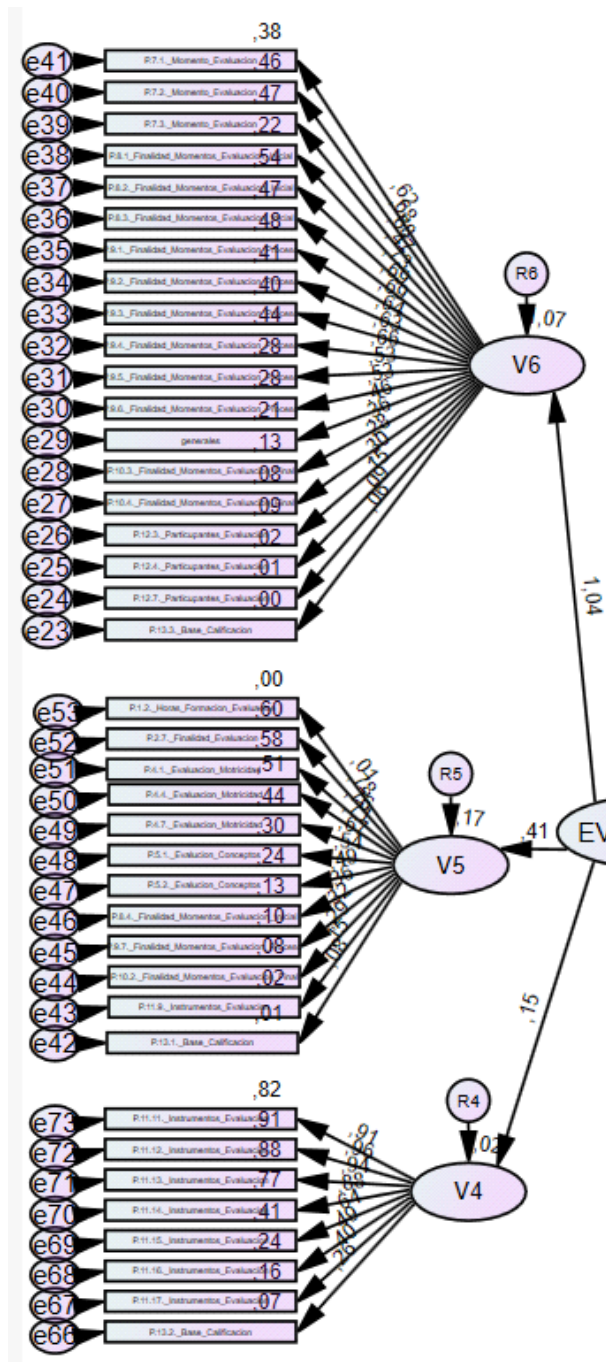

Figura 1. Resultados Análisis Factorial Confirmatorio. ajuste adecuado para el modelo (González-M ontesinos y Backhoff, 2010; Grao-Cruces, Teva-Villén, PérezO rdás y Blanco-Luengo, 2016; Pérez y M edrano, 2010) mostrando que la estructura propuesta es válida para esta escala.

\section{Consistencia Interna}

El valor del Alpha de Cronbach para la escala gl obal hasido de $\alpha=.94$, un excelenteíndice de fiabilidad (FríasNavarro, 2019; Nunnally, 1978). Todos los elementos de la escala correlacionan positivamente y con valores relativamente buenos con el total de la escala (Tabla 5). Ú nicamente el ítem 11.8. con un $r=.098$ obtiene un valor más bajo. Se toma la decisión de mantener ese ítem por el valor que tiene para profundizar en los aspectos en los que se centra la investigación. Además, como se observa en laTabla 5 ningún elemento muestra que mejore el valor de $\alpha$ si se elimina el elemento.

Igualmente, se comprobó el valor de Alpha de Cronbach independientemente para la escal a correspondiente a los niveles educativos de Educación Primaria y
00

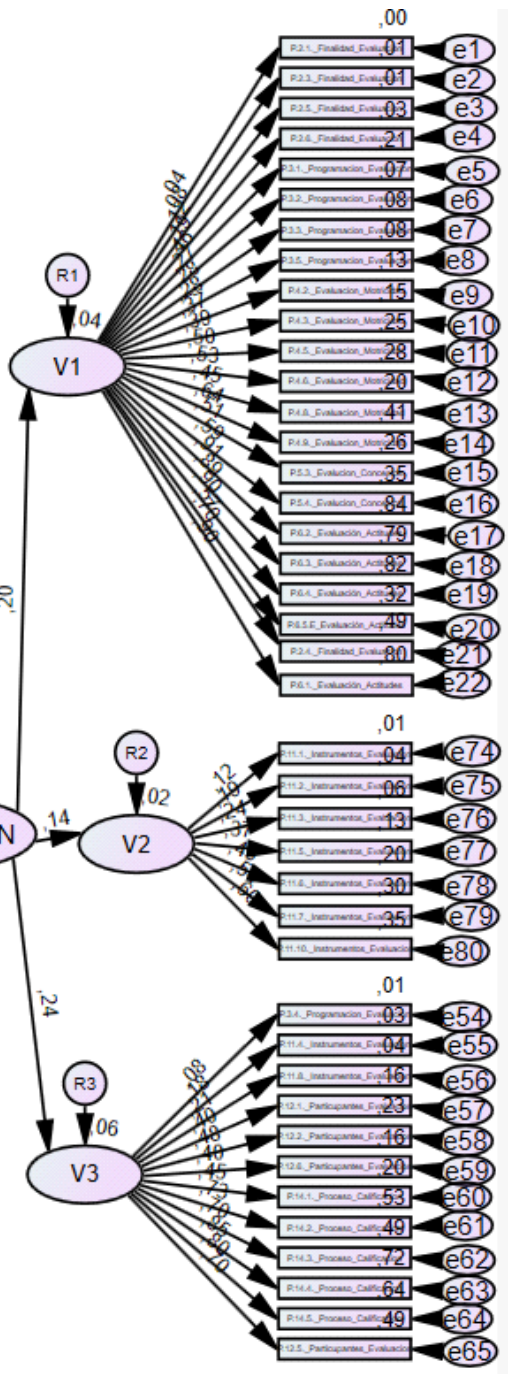

Educación Secundaria 0 bligatoria, danto valores de $\alpha=.95$ y $\alpha=.92$ respectivamente. Por 10 tanto, puede concluirse que la escala presenta buenos valores de fiabilidad tanto en el conjunto global como por cada nivel educativo.

\section{Correlaciones en-} tre las dimensiones de la escala

Se analizan las correlaciones entre $13 \mathrm{di}-$ mensiones de la escala para comprobar la asociación entre las mismas (tabla6). Paraello seutiliza el coeficiente de correlación de Pearson. Todas las dimensiones correlacionan medianay positivamente $(r=.331$; $\mathrm{p}<.005)$ mostrando dimensiones próximas en el continuo. Las dimensiones que muestran 
Tabla 6.

Correlaciones entre las dimensiones de la escal

\begin{tabular}{|c|c|c|c|c|c|c|c|c|c|c|c|c|c|}
\hline & 1 & 2 & 3 & 4 & 5 & 6 & 7 & 8 & 9 & 10 & 11 & 12 & 13 \\
\hline 1. Finalidad & 1 & $.627 * *$ & $.705^{* *}$ & $.527^{* *}$ & $.607 * *$ & $.254^{* *}$ & $.375^{* *}$ & $.474 * *$ & $.472^{* *}$ & $.262^{* * *}$ & $.225^{* *}$ & $.300^{* *}$ & $.137^{* *}$ \\
\hline 2. Programación & - & 1 & .551 & $.460^{* *}$ & $.505 * *$ & $.356 * *$ & $.355 * *$ & $.455 * *$ & $.358 * *$ & $.278^{* *}$ & $.304 * *$ & $.182 * *$ & $.280^{* *}$ \\
\hline 3. Evaluación Motricidad & - & - & 1 & $.587 * *$ & $.585^{* *}$ & $.236 * *$ & $.399 * *$ & $.407 * *$ & $.463 * *$ & $.342^{* *}$ & $.235 * *$ & $.252 * *$ & $.169 * *$ \\
\hline 4. Evaluación Conceptos & - & - & - & 1 & $.385^{* *}$ & $.194 * *$ & $.372^{* *}$ & $.302 * *$ & $.307 * *$ & $.310^{* *}$ & $.225^{* *}$ & $.203 * *$ & $.183^{* *}$ \\
\hline 5. Evaluación Actitudes & - & - & - & - & 1 & $.298 * *$ & $.280^{* *}$ & $.308 * *$ & $.371^{* *}$ & $.110^{*}$ & $.214^{* *}$ & $.238 * *$ & $.162^{* *}$ \\
\hline 6. Momentos & - & - & - & - & - & 1 & $.471^{* *}$ & $.418 * *$ & $.329 * *$ & $.197 * *$ & $.422 * *$ & $.044^{*}$ & $.242^{* *}$ \\
\hline 7. Evaluación inicial & - & - & - & - & - & - & 1 & $.530 * *$ & $.441 * *$ & $.364 * *$ & $.320 * *$ & $.318^{* *}$ & $.335^{* *}$ \\
\hline 8. Evaluación Proceso & - & - & - & - & - & - & - & 1 & $.573 * *$ & $.264^{* *}$ & $.335^{* *}$ & $.270^{* *}$ & $.242^{* *}$ \\
\hline 9. Evaluación Final & - & - & - & - & - & - & - & - & 1 & $.229 * *$ & $.164 * *$ & $.326^{* *}$ & $120 * *$ \\
\hline 10. Instrumentos & - & - & - & - & - & - & - & - & - & 1 & $.246 * *$ & $.314^{* *}$ & $.256 * *$ \\
\hline 11. Participantes & - & - & - & - & - & - & - & - & - & - & 1 & $.118^{*}$ & $.611^{* *}$ \\
\hline 12. Base Cal ificación & - & - & - & - & - & - & - & - & - & - & - & 1 & $.161^{* *}$ \\
\hline 13. Proceso Calificación & - & - & - & - & - & - & - & - & - & - & - & - & 1 \\
\hline
\end{tabular}

importante al área de la evaluación en $E F$, ya que son escasos los estudios previos que desarrollen un cuestionario de características similares. La amplitud del cuestionario diseñado permite abordar en un único instrumento un gran número de dimensiones sobre los procesos de evaluación de gran relevancia para conocer la percepción de los docentes sobre el

mejores valores de correlación son Finalidad y Evalua ción de contenidos motrices $(r=.705 ; p<.001)$, y, Fina lidad y Programación ( $r=.627 ; p<.001)$, mientras que las dimensiones de momentos de evaluación y base de calificación presentan valores de correlación muy bajos $y$ sin significatividad ( $r=.044 ; p>.005)$.

\section{Discusión y conclusiones}

El Cuestionario «\# EvalEF» sobre Procesos de Evaluación en Educación Física ha mostrado ser un instrumento válido y fiable para recoger información sobre la percepción de los docentes de los procesos de evaluación que llevan a cabo en sus clases de EF. Los resultados han mostrado niveles adecuados de fiabilidad y validez factorial. La consistencia interna de la escala muestra valores muy buenos, tanto de forma global, como por niveles educativos.

Los resultados muestran una estructura de seis factores que concuerda con al gunas de las dimensiones planteadas en los estudios desarrollados por Rodríguez-Negro y Zulaika (2016) y González-Palacio et al. (2021), aunque el cuestionario de estos últimos recoge la percepción de los estudiantes. Las dimensiones del cuestionario propuesto en esta investigación profundizan en mayor media en los procesos de evaluación docente recogiendo la percepción de 469 docentes de 15 Comunidades Autónomas, siendo una muestra bastante más amplia que la de los estudios previamente menciona dos. Por otra parte, aunque se encuentran varios estudios que analizan la contribución de la evaluación formativa en el ámbito universitario desde el punto de vista de los docentes (De la O rden Hoz y Pimienta Prieto, 2016) y el alumnado (López-Pastor y PalaciosPicos, 2015; Castejón-O liva et al., 2015) a través de un cuestionario, la información obtenida no puede contrastarse con la del presente estudio, por la diferencia de las características de los procesos de evaluación desarrolla dos en estas etapas.

Por ello, este cuestionario realiza una aportación

Retos, número 42, 2021 (40 trimestre) proceso de evaluación que desarrollan en el aula. Esto nos permite identificar cuáles son los factores que el profesorado tiene más en cuenta a la hora de realizar la evaluación, obteniendo una visión general sobre el modelo más empleado y proporcionando una visión amplia acerca de avances en este aspecto. El instrumento diseñado puede ser de utilidad para comprobar en qué aspectos puede ser necesario incidir más en la formación inicial y permanente de los docentes para conseguir verdaderos cambios en los procesos de evaluación que se desar rollan. A un nivel más práctico e incluso de mejora de la propia práctica docente, este cuestionario puede resultar de utilidad para el propio profesorado de EF, ya que les puede ayudar a ser más conscientes de su propio proceso de evaluación y a valorar opciones de mejora dentro de su propia práctica.

Esta investigación también presenta ciertas limitaciones. Entre ellas, encontramos que el cuestionario no está diferenciado por etapas (Primaria vs. Secundaria) lo que puede hacer que al gunas características propias de la etapa educativa no estén recogidas. También, no haber podido obtener una muestra representativa de todas las Comunidades Autónomas que conforman el territorio nacional. Por otra parte, el hecho de ser información auto-reportada por los docentes no nos permite aseverar que las respuestas proporcionadas coincidan con la práctica real, al igual que no podemos analizar en profundidad el contexto específico en el que los docentes participantes ejercen su labor profesional, ni conocer los motivos por los que los docentes deciden realizar la evaluación de una manera u otra. Por otro lado, el escaso número de estudios de características similares encontrados dificultan la realización de una comparación y discusión de resultados, lo que a su vez destaca la importancia que puede tener el estudio en el ámbito de la EF. Por último, entre las líneas futuras de investigación seencuentra, principalmente, emplear este cuestionario para recoger información del contexto español que nos permita tener una visión general de cómo se desarrollan los procesos de evaluación en el aula y empezar a realizar propuestas concretas para avanzar en este campo de estudio. 


\subsection{6/ rimcafd2015.58.004}

Cervantes, V.H. (2005). Interpretaciones del coeficienteAlpha de Cronbach. Avances en Medición, 3, 9-28.

Chaverra, Fernández, B.E. , \& Hernández Álvarez, J.L. (2019). La acción evaluativa en Profesores de Educación Física: Una investigación M ulti-Casos. Revista Iberoamericana deEvaluación Educativa, 12(1), 211- 228. https: / / doi.org/ 10.15366/ riee2019.12.1.012

Chng, L.S. \& Lund, J. (2018). Assessment for Learning in Physical Education: The what, why and how. Journal of Physical Education, Recreation \& Dance, 89 (8), 29-34. https: / / doi.org/ 10.1080/ 07303084.2018 .1503119

De la Orden, A. \& Pimienta-Prieto, J.H. (2016). Instrumento para determinar los tipos de evaluación utilizados por los profesores universitarios. Revista Electrónica de Investigación Educativa, 18(2), 40-52.

Díaz-Lucea, J. (2012). La evaluación formativa como instrumento de aprendizaje en Educación Física. Barcelona: INDE.

Frías-N avarro, D. (2019). Apuntes de consistencia interna de las puntuaciones de un instrumento de medida. Universidad deValencia. España. Disponible en: https:/ / www.uv. es/ friasnav/ AlfaCronbach. pdf

Garrido-Guzmán, M. E. , Zagalaz-Sánchez, M. L., TorresLuque, G. \& Romero-Granados, S. (2010). Diseño y validación de un cuestionario para técnicos deportivos acerca de su opinión de las actitudes de padres y madres en el deporte (CTPMD). Cuadernos de Psicología del deporte, 10(2), 39-48. https:// revistas. um.es/cpd/ article/ view/ 113001

Gil-Flores, J. \& García Iiménez, E. (2014). Evaluación formativa y resultados de aprendizaje en los centros que imparten Educación Secundaria O bligatoria. Revista Española de Pedagogía, 72, 437-455.

González-M ontesinos, M.J. \& Backhoff, E. (2010). Validación de un cuestionario de contexto para evaluar sistemas educativos con model os de Ecuaciones Estructurales. Revista Electrónica de Investigación y Evaluación Educativa, 16(2), 1-17. http:/ / www. uv.es/ RELIEVE/ v16n2/ RELIEVEv16n2_1. htm

González-Palacio, E.V., Chaverra-Fernández, B.E., Bustamante-Castaño, S.A. \&Toro-Suaza, C.A. (2021). Diseño y validación de un cuestionario sobre las concepciones y percepción de los estudiantes sobre la evaluación en educación física. Retos, 40, 317-325. https:/ / doi.org/ 10.47197/ retos. v1i40.80914 https: / / doi.org/ 10.47197/ retos. v1i40.80914

Grao-Cruces, N.A., Teva-Villén, A., Pérez-0 dás, MR. \& Blanco-Luengo, D. (2016).Validez de constructo de la escala motivos de abandono de centros deportivos. Revista internacional de Medicina y Ciencia de la Actividad Física y el Deporte, 16(61), 1-15. https:/ / doi.org/ 10.15366/ rimcafd2016.61.001

Herrero-González, D. , M anrique-Arribas,J.C. \& LópezPastor, V.M. (2021). Incidencia de la Formación inicial y permanente del profesorado en la aplicación de la evaluación formativa y compartida en educa ción física. Retos, 41, 533-543. https:/ / doi.org/ 10.47197/ retos. v0i41.86090

Jiménez-J iménez, F. \& N avarro-A delantado, V. (2008). Evaluación formativa y metaevaluaicón en Educación Física: Dos estudios de casos colectivos en las Etapas de Educación Primaria y Secundaria. Revista española de Educación Física y D eporte, 9, 15-25.

López-Pastor, V.M. (2005). La evaluación como sinónimo de calificación. Implicaciones y efectosen laEducación y en la Formación del Profesorado. Revista electrónica interuniversitaria de formación del profesorado, 8(4), 1-7. ttp:/ / www. aufop. com/ aufop/ home/ López-Pastor, V.M . (2006a). La evaluación en Educación Física. Revisión de modelos tradicionales y plantea mientos de una alternativa. La evaluación formativa y compartida. Retos, Nuevas tendencias en Educación Física, D eportey Recreación 10, 31-41. https:/ / doi.org/ 10.47197/ retos. v0i10.35061

López-Pastor, V.M. (2006b). El papel de la evaluación formativa en el proceso de convergencia hacia el E.E.E.S. Análisis del estado de la cuestión y presentación de un sistema de intervención. Revista Interuniversitaria deFormacion del Profesorado, 20(3), 93119.

López-Pastor, V.M . (2013). Nuevas perspectivas sobre Evaluación en Educación Física. Revista de Educación Física, 29(3), 4-13.

López-Pastor, V. M. (2017). Evaluación formativay compartida: evaluar para aprender y la implicación del alumnado en los procesos de evaluación y aprendizaje. En V.M. López-Pastor \& Á. Pérez-Pueyo (Coords). Evaluación formativa y compartida en educación: experiencias de éxito en todas las etapas educativas (pp. 34-68). León: U niversidad de León.

López-Pastor, V.M., Barba-Martín, J.J., M onjas-Aguado, R., Manrique-Arribas, J.C., Heras-Bernandino, C., González-Pascual, M. \& Gómez-García, J.M. (2007). Trece años deevaluación compartidaen Educación Física. Revista Internacional de M edicina y Ciencias de la Actividad física y del Deporte, 7(26), 69-86. http:/ / cdeporte. rediris. es/ revista/ revista26/ artautoeval $48 . h \mathrm{hm}$ 
López-Pastor, V.M . \& Palacios-Picos, A. (2015). Percepción de los futuros docentes sobre los sistemas de evaluación de sus aprendizajes. Revista Teórica de la Educación: Educación y Cultura en la Sociedad de la información, 13(3), 317-341.

Ní-Chróniín, D. \& Cosgrave, C. (2013). Implementing formative assessment in primary physical education: teacher perspectives and experiences. Physical Education and sport Pedagogy, 18 (2), 219-233. http:/ / dx.doi.org/ 10.1080/ 17408989.2012.666787

N unnally, J. (1978). Psychometric theory. M cGraw-Hill

Pérez, E.R. \& Medrano, L. (2010). Análisis Factorial Exploratorio: Bases Conceptuales y M etodológicas. Revista Argentina de Ciencias del Comportamiento, 2(1), 58-66.

Rodríguez-N egro, J., \& Zulaika, L.M. (2016). Evaluz ción en educación Física. Análisis comparativo entre la teoría oficial y la praxis cotidiana. Sportis. Revista técnico- Científica del Deporte Escolar, Educación Física y Psicomotricidad, 2(3), 421-438.http:/ / dx.doi.org/ 10.17979/ sportis. 2016.2.3.144

Rodríguez-García, P.L. (2006). Educación Física y Salud en Primaria. Hacia una educación corporal significativa y autónoma. Barcelona: Inde.

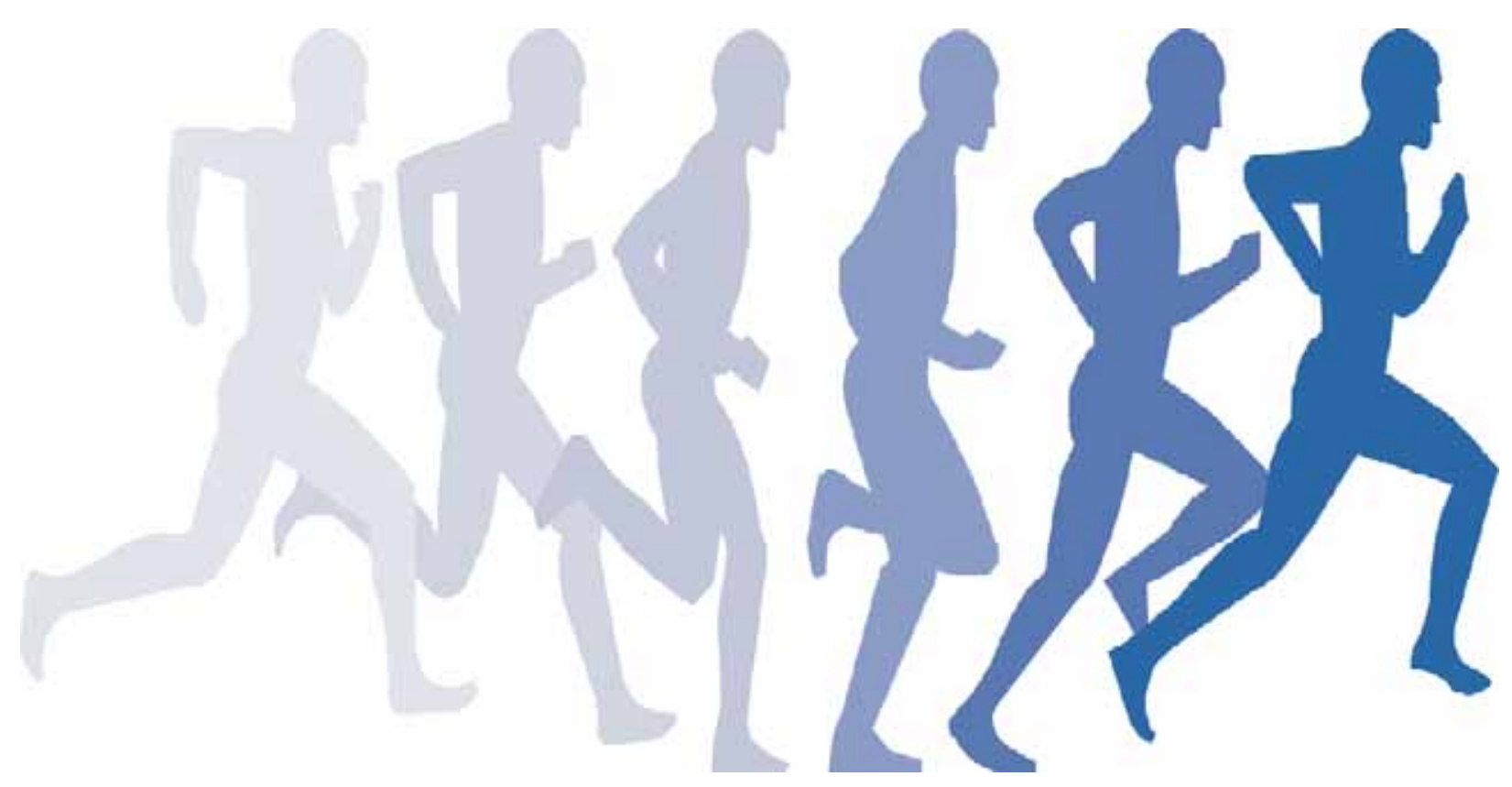

